\title{
Clinical Olfactory Working Group Consensus Statement on the Treatment of Post Infectious Olfactory Dysfunction
}

Alfred B Addison ${ }^{1}$, Billy Wong ${ }^{1}$, Dr Tanzime Ahmed ${ }^{1}$, Alberto Macchi ${ }^{2}$, Iordanis Konstantinidis ${ }^{3}$, Caroline Huart ${ }^{4,5}$ Johannes Frasnelli ${ }^{6,7}$, Alexander W. Fjaeldstad ${ }^{8,9}$, Vijay R. Ramakrishnan ${ }^{10}$, Philippe Rombaux ${ }^{4,5}$, Katherine L Whitcroft ${ }^{11,12}$ Eric H. Holbrook ${ }^{13}$, Sophia C. Poletti ${ }^{14}$, Julien W. Hsieh $^{15}$; Basile N. Landis ${ }^{15}$, James Boardman ${ }^{16}$, Antje Welge-Lüssen ${ }^{17}$, Devina Maru ${ }^{18}$, Thomas Hummel $^{19}$, Carl M. Philpott ${ }^{16,20,21}$

1. East Sussex North Essex Foundation Trust, Heath Road, Ipswich, IP4 5PD, UK

2. ENT University of Insubria, Italian Academy of Rhinology, Asst Sette-Laghi, Varese, Italy

3. Smell and Taste Clinic, 2nd ORL University Department, Aristotle University, Thessaloniki, Greece

4. Department of Otorhinolaryngology, Cliniques Universitaires Saint-Luc, Brussels, Belgium

5. Institute of Neuroscience, Université Catholique de Louvain, Brussels, Belgium

6. Department of Anatomy, Université du Québec à Trois-Rivières, Trois-Rivières, QC, Canada

7. Research Center, Sacré-Coeur Hospital Montréal, Montréal, QC, Canada

8. Flavour Clinic, ENT Department, Regional Hospital West Jutland, Holstebro, Denmark

9. Flavour Institute, Aarhus University, Aarhus, Denmark

10. Departments of Otolaryngology and Neurosurgery, University of Colorado Anschutz Medical Campus, Aurora, CO 80045, USA

11. Centre for the Study of the Senses, Institute of Philosophy, School of Advanced Study, London

12. South Yorkshire Deanery, Yorkshire and Humber School of Surgery, UK

13. Department of Otolaryngology-Head and Neck Surgery, Massachusetts Eye and Ear, Harvard Medical School

14. Department of Otorhinolaryngology - Head and Neck Surgery, Inselspital, Bern University Hospital, University of Bern, Bern, Switzerland

15. Rhinology-Olfactology Unit; Department of Otorhinolaryngology, University Hospitals of Geneva, Geneva, Switzerland

16. Fifth Sense, Barrow-in-Furness, Cumbria, UK

17. Department of Otorhinolaryngology, University Hospital Basel, University of Basel, Basel, Switzerland

18. Royal College of General Practitioners, London, UK

19. Smell \& Taste Clinic, Department of Otorhinolaryngology, TU Dresden, Dresden, Germany

20. The Norfolk Smell \& Taste Clinic, James Paget University Hospital NHS Foundation Trust, Gorleston, NR31 6LA, UK

21. Norwich Medical School, University of East Anglia, Norwich, NR4 7TJ, UK

Corresponding Author

Professor Carl Philpott

Norwich Medical School,

University of East Anglia,

Norwich,

NR4 7TJ,

UK

01603591105

C.Philpott@uea.ac.uk

All authors have no conflict of interest to disclose. 


\section{Content table}

1. Abstract

2. Introduction

3. Evaluation of olfactory dysfunction

4. Systematic review of literature

5. Survey of experts

6. Practical challenges to management

7. Practical advice for general practitioners

8. Practical advice for otolaryngologists

9. Conclusion 


\section{Abstract}

Background: Respiratory tract viruses are the second most common cause for olfactory dysfunction. As we learn more about the effects of the severe acute respiratory syndrome coronavirus (SARSCoV-2), with the recognition that olfactory dysfunction is a key symptom of this disease process, there is now a greater need for evidence-based management for post infectious olfactory dysfunction (PIOD).

Objective: To provide an evidence based practical guide to the management of PIOD (including postcovid cases) for both primary care practitioners and hospital specialists.

Methods: A systematic review was performed on the treatment options available for the management of PIOD. The written systematic review was then circulated among the members of the Clinical Olfactory Working Group (COWoG) for their perusal prior to round table expert discussion of the treatment options. The group also undertook a survey to determine their current clinical practice with regards to treatment of PIOD.

Results and Conclusions: The COWoG members made an overwhelming recommendation for olfactory training and none recommended monocycline antibiotics. The diagnostic role of oral steroids is discussed and there were some in favour of vitamin A drops. Further research is needed to confirm the place of other therapeutic options. 


\section{Clinical Implications}

Patients with COVID-19 and other infection related olfactory dysfunction should be guided through olfactory rehabilitation and be signposted to specialists for other treatments in refractory cases.

\section{Capsule Summary}

To date there have not been any definitive recommendations on how to treat post-infectious olfactory dysfunction. This article summarises the evidence and provides expert consensus on how to apply this in practice.

Keywords: olfaction, olfactory disorders, viral infections, hyposmia, anosmia, parosmia therapy, COVID-19.

\section{Glossary}

ACE

BTT

Angiotensin converting enzyme

CCCRC butanol threshold testing

CCSIT

Connecticut Chemosensory Clinical Research Centre test

COWoG

Cross-Cultural Smell Identification Test

(M)OT

$\mathrm{OB}$ Clinical Olfactory Working Group

PIOD (modified) olfactory training

PTOL Olfactory bulb

$\mathrm{T} \& \mathrm{~T}$ Post-infectious olfactory dysfunction (AKA post-viral olfactory loss/dysfunction)

UPSIT Post-traumatic olfactory loss

URTI Toyota \& Takagi olfactometer 


\section{Introduction}

Loss of smell is a common complaint in adults and yet has been underestimated ${ }^{1}$. Anosmia, complete loss of smell, is thought to affect at least $1 \%$ of the population with the overall estimated prevalence of olfactory disorders now thought to be over $20 \%{ }^{2-4}$. Upper respiratory tract infections are usually associated with decreased smell during their acute phase ${ }^{5-7}$. Post-infectious olfactory dysfunction $\underline{\text { (PIOD) }}$ represents an important frequent cause of persistent olfactory dysfunction ${ }^{1}$, accounting for $11 \%$ of all causes ${ }^{8}$ but $20-30 \%$ of cases in specialized smell and taste clinics ${ }^{9,10}$ and typically (preCOVID) affecting women over 50 years of age. Olfaction plays important roles in daily life, ranging from safety perception (ability to detect hazardous substances, fire and rotten foods) to psychosocial functions (such as recognition of kin or emotions mediated through body odours) and enjoyment of food and drink ${ }^{11}$. Olfactory dysfunction may therefore lead to significant morbidity in the form of nutritional disturbance, social anxiety or depression ${ }^{11-13}$.

The emergence of the severe acute respiratory syndrome coronavirus 2 (SARS-CoV-2) pandemic with its devastating global effects has brought public awareness to the impact of viral infections on olfactory function ${ }^{14,15}$. There is now an emerging cluster of new patients with PIOD - those infected with the SARS-CoV-2 virus as part of the pandemic. With smell loss now an official World Health Organisation symptom of COVID-19, it is estimated that over $60 \%$ of those contracting the virus are affected by this symptom ${ }^{16-19}$, including those who are otherwise asymptomatic ${ }^{20}$. Extrapolating from this figure, it is therefore possible that nearly 20 million people globally will have experienced smell loss due to COVID-19 by October 2020. COVID-19 infection appears to have a different pattern of smell loss than typical PIOD cases with a more profound loss of smell and with taste loss, specifically bitter loss, identified as a discriminating feature ${ }^{21}$. In another study SARS-CoV-2 affected mainly odour thresholds, possibly suggesting that the major cause of loss of smell lies at the level of the olfactory neuroepithelium, rather than in the central nervous system ${ }^{20}$. Current thinking around the mechanism of chemosensory dysfunction in COVID-19 infection suggests that it is caused by viral entry via ACE2 receptors, with infection and death of sustentacular cells in the olfactory epithelium; this does not necessarily lead to infection, damage, death, and the need for regeneration of olfactory receptor neurons in the majority of cases and hence why they appear to recover within 4 weeks of the onset $^{22}$. The current data suggest that $10-17 \%$ have continued smell loss (i.e. COVID-19-related PIOD) and do not recover spontaneously ${ }^{23,24}$. Notably, many of these patients report parosmia during recovery ${ }^{25}$. The reasoning for the persistence of OD in these cases may be attributed to a larger area of the olfactory epithelium being affected by the coronavirus, possibly with cellular death of a larger number of olfactory receptor neurons; further evidence is required to support this theory ${ }^{22}$. The current understanding and pathogenesis of non-COVID-19 olfactory dysfunction following a 
viral upper respiratory tract infection begins with inflammation of the nasal mucosa as a result of the acute infection. This disrupts the natural airway conduction through the nasal cavity hence inhibiting the transport of odorants to the olfactory epithelium. Impaired olfaction may also result from dysfunction at the level of the olfactory receptor neuron, though the precise pathophysiological mechanism is virus dependant. Rhinoviruses, for instance, cause a selective neutrophil and monocyte recruitment to occur. The inflammatory cascade that ensues includes an increase in bradykinin, cytokine, chemokine and sICAM-1 concentrations ${ }^{26}$. The response in an immunocompetent individual involves T-lymphocyte activation allowing the viral pathogen to be eliminated. Viral pathogens that have been implicated in PIOD other than rhinoviruses include parainfluenza virus and Epstein-Barr virus with the frequency of virus found in studies varying ${ }^{27}$; for example, parainfluenza accounted for $88 \%$ in one study ${ }^{28}$,

With specific respect to the olfactory apparatus, these viruses appear to cause some partial loss of olfactory receptor neurons in the olfactory epithelium. Ultrastructurally, studies have revealed a markedly disorganised epithelium with decrease in the number of olfactory receptor cells and nerve bundles with squamous metaplasia occurring in a few cases ${ }^{29}$. This reduction in the number of olfactory receptor neurons means at the epithelial surface there is a lack of dendrites and vesicles, therefore, a decrease in the area available for odour molecule detection ${ }^{30}$. Jafek et al reported that especially in patients with anosmia the few dendrites that were present did not reach the epithelial surface and appeared shrunken ${ }^{31}$. Patients with PIOD have been also found to have reduced olfactory bulb volume and patchy distribution of neuroepithelia perhaps due to the process of remodelling and replacement ${ }^{32}$. Calculation of the olfactory bulb (OB) volume studied through MRI scanning in coronal T2 sequences demonstrates an overall reduced OB volume on both sides even if normalized to age and sex. OB volume above $40 \mathrm{~cm}^{3}$ for one olfactory bulb shows a trend for a good prognosis for recovery. This must be considered in conjunction with the age, the sex of the patient, the duration between the onset of the symptoms and the period when the test has been made and to other potentials toxins the patient would have been exposed to ${ }^{32}$.

The prognosis of PIOD is variable; it has been suggested that up to a third of patients presenting at specialised, tertiary smell and taste clinics undergo spontaneous recovery over a period of 12-18 months ${ }^{33}$. The presence of parosmia has recently been demonstrated to be associated with a more favourable prognosis for recovery ${ }^{34}$. Different treatment modalities have been tried, ranging from smell training through to a variety of medical treatments, both topical and systemic ${ }^{35}$. This consensus document aims to combine the collective experience of the COWoG with the current evidence base to establish guidance for medical and non-medical treatments for patients with PIOD. 


\section{Evaluation of Olfactory Dysfunction}

In general, evaluation of olfactory dysfunction can be performed by three different methods; subjective, patient reported assessment; psychophysical testing; and less biased measures such as olfactory event-related potentials or functional magnetic resonance imaging.

Subjective assessment is performed using various methods including, visual analogue scales and questionnaires. Although olfactory specific questionnaires such as the Questionnaire for Olfactory Dysfunction can be used to differentiate between normosmia and hyposmia, they tend to be unreliable compared with psychophysical testing and are best used to assess the impact on quality of life ${ }^{36}$. Subjective testing can be a valuable tool in monitoring the clinical effect of treatment and therefore should be used in conjunction with other objective testing ${ }^{1}$. Ideally only validated questionnaires should be used when assessing patients with olfactory dysfunction and these should be used with other psychophysical tests, given their lack of accuracy when used alone ${ }^{36}$.

Apart from olfactory testing, clinical evaluation should always include nasal endoscopy focusing on the patency of olfactory cleft and the presence of findings suggesting nasal inflammation such as polyps, turbinate hypertrophy, oedema, purulent secretions etc. Assessment in this way should ideally be quantified using a validated scoring system, such as the Lund-Kennedy or Olfactory Cleft Endoscopy scale ${ }^{37}$. In a certain percentage of patients with PIOD an underlying inflammatory process in the nose may already exist and its recognition, if not already diagnosed, could modify the treatment options. Obviously, this has been problematic during the COVID-19 pandemic when face-to-face contact and nasal endoscopy have been avoided where possible. Measures may need to be taken to screen patients for COVID-19 to reduce the potential for transmission of infection to the healthcare team. Where any doubt regarding COVID-19 status exists, appropriate personal protective equipment should be worn.

Psychophysical assessment of olfactory function employs the use of an olfactory stimulus presented to the patient and the outcome of the test is dependent on the patient's response. It requires a patient who is cooperative, understands and follows instructions ${ }^{1}$. Different aspects of olfactory testing including threshold and suprathreshold assessment can be performed. Odour threshold is the lowest concentration of an odorant detectable to a participant and does not require odour identification ${ }^{38}$. Odour discrimination describes the non-verbal ability to differentiate between different odours. Odour identification involves both recognition of a stimulus and communication of its correct identity (i.e., the ability to name an odour from a list of descriptors). Odour identification requires previous 
exposure to odour stimulus, and may therefore be culturally specific, hence these tests need adapting for different communities ${ }^{1}$. The usefulness of testing for multiple psychophysical components of olfaction (e.g. threshold, discrimination and identification) when assessing olfactory dysfunction is debated. There is however good evidence that both odour threshold and supra threshold testing adds to the diagnostic value of psychophysical assessment ${ }^{39}$; odour threshold detection may be more affected by COVID-19 compared to odour identification ${ }^{20}$.

There are various tests commercially available which measure either just one aspect of olfaction or multiple components, such as the Sniffin' Sticks test (Burghart, Germany) ${ }^{40,41}$, Smell Diskettes (Novimed, Switzerland) ${ }^{42}$ and the University of Pennsylvania Smell Identification Test (Sensonics, USA) ${ }^{43}$; other non-commercial tests have also been validated such as the Connecticut Chemosensory Clinical Research Centre Test (USA) ${ }^{44}$ and the Toyota \& Takagi (T\&T) olfactometer (Japan) ${ }^{45}$. The U-Sniff has been validated for children ${ }^{46,47}$ and the European retronasal test for smell perception when eating 48 .

Retronasal olfaction can be tested by asking patients to identify flavoured powders. Such tests are useful where there is diagnostic uncertainty. For example, it has been demonstrated that in cases of sudden onset olfactory dysfunction, such as posttraumatic loss, both orthonasal and retronasal functions decline concurrently. However, more progressive dysfunction, such as is seen in sinonasal disease, may preferentially affect the orthonasal route whilst retronasal olfaction may be preserved ${ }^{49}$, ${ }^{50}$. As part of a full olfactory assessment, screening of gustatory function should be undertaken. This can be achieved using liquids applied to the tongue for sweet, salty, sour or bitter (umami is not commonly screened for as it is poorly identified).

Electrophysiological studies include electroencephalography (EEG) and electro-olfactograms (EOG - the recording of generator potentials via an electrode in contact with the olfactory neuroepithelium). As EEG and EOG are both event-related, delivery of a known concentration of odorant must be precisely controlled using an olfactometer, which requires certain equipment and specific technical expertise and this limits the use of such testing for routine clinical purposes ${ }^{51}$.

MRI scanning allows for calculation of olfactory bulb volume, olfactory sulcus depth, and upstream volumetric assessment of olfactory eloquent regions all of which have been linked to olfactory function ${ }^{52,53}$. In addition, CT and MRI provide information regarding inflammation of nasal and paranasal mucosa in cases with suggestive history of concomitant inflammatory pathology where endoscopy was not conclusive. They may also reveal evidence of central pathology contributing to 
smell dysfunction, such as sequelae of prior head trauma or microvascular disease. Functional MRI can provide dynamic assessment of olfactory related cortical activity but is limited in its use in individual patients ${ }^{54}$.

\section{Literature Review}

A comprehensive electronic database search based on the updated guidelines for systematic reviews of the Cochrane Collaboration Review Group ${ }^{55}$ and the Preferred Reporting Items for Systematic Reviews and Meta-Analysis (PRISMA) was performed ${ }^{56}$. The population, intervention, comparisons, outcomes and study design (PICOS) algorithm guided data extraction ${ }^{57}$.

On 6 July 2020, a systematic search of electronic databases (PubMed, Google Scholar, Cochrane database, Web of Science, Scopus and Embase) was conducted. Using the Cochrane methodological filter for randomised control trials (RCTs) in addition to combing MeSH keywords and other relevant terms including, “anosmia", "hyposmia”, “dysosmia”,"parosmia”, “pharmacotherapy”, “olfactory dysfunction", "post-viral”, "post-infectious", "olfactory impairment", "olfactory disturbance”, “olfactory loss", "smell disorder", "viral infection", "virus", "viral disease", “common cold”, and "respiratory tract infection," were searched to identify primary comparative studies on treatment and management options for post viral olfactory loss. Further cross screenings of the references were performed to complement initial search results. Comparative studies of any design examining the management outcome of patients with post viral olfactory loss were included.

The level of evidence was established for each publication and the risk of bias was analysed for each study included. The Modified Cochrane Collaboration Tool for Assessing Risk of Bias was applied to level 1 and 2 studies and The Newcastle-Ottawa Quality Assessment Scale was applied for studies in the level 3 and 4 categories. By following the modified GRADE quality assessment; the quality of evidence for the treatment option was graded and recommendations (table 2) were provided when there was sufficient high-level evidence for a particular intervention. Each subsequent author reviewed, critiqued and amended the recommendations and any discrepancies between the authors were discussed until a consensus was reached.

\section{Inclusion Criteria}

All published studies on treatment of PIOD including:

- Randomised Control Trials

- Cohort studies 
- Preliminary results of ongoing research

\section{Exclusion Criteria}

- Case reports/ letters to the editor/and book chapters

- Non-English publications

- Study subjects with olfactory loss secondary to aetiologies not including PIOD (e.g. post traumatic, congenital, etc)

\section{Results}

The search resulted in 467 citations from which the relevant studies were selected for review and duplicates were removed. In total, 107 articles were fully reviewed and analysed for eligibility; 40 citations fulfilled the inclusion criteria, 11 of which were randomised controlled trials. In total, 15 of the articles specifically looked at PIOD whilst the other 25 included other aetiologies for olfactory dysfunction. All the articles were human studies primarily related to the outcomes of management in patients with PIOD (see flow chart - figure 1)

The combined total number of patients included in this review were 4983 patients; 2352 (47.2\%) patients had PIOD. The patients with PIOD were diagnosed based on self-reported history of a preceding (viral) upper respiratory tract infection (URTI). A diagnosis of PIOD should be made in line with international guidelines ${ }^{58}$. The most commonly used test kit was the Sniffin' Sticks ( $n=18$ studies). Other tests utilised included UPSIT, Toyota \& Takagi olfactometer (T\&T), Cross-Cultural Smell Identification Test (CCSIT), Connecticut Chemosensory Clinical Research Centre (CCCRC) test, butanol threshold testing (BTT), and a Visual Analog Scale (VAS) and/or additional subjective scales. 
Table 1: Post Infectious Olfactory Dysfunction Clinical Trials

\begin{tabular}{|c|c|c|c|c|c|c|c|}
\hline \multicolumn{8}{|c|}{ Olfactory Training (OT) } \\
\hline Study & Study Design & Patients /Aetiology & $\begin{array}{l}\text { Olfactory function } \\
\text { Test }\end{array}$ & Intervention & Follow up (weeks) & Results & Level of evidence \\
\hline Choi et al, $2020^{59}$ & Prospective controlled & $\mathrm{PIOD}=104$ & $\begin{array}{l}\text { Korean version of } \\
\text { Sniffin' Sticks }\end{array}$ & Olfactory Training & 12 & $\begin{array}{l}\text { Significant } \\
\text { improvement in } \\
\text { threshold and } \\
\text { identification in OT } \\
\text { group. No change in } \\
\text { discrimination }\end{array}$ & 3 \\
\hline Kim et al,2018 ${ }^{60}$ & Prospective case series & $\mathrm{PIOD}=82$ & BTT and CCSIT & $\begin{array}{l}\text { Korean odorants for } \\
\text { OT }\end{array}$ & 24 & $\begin{array}{l}\text { Improved BTT and } \\
\text { CCSIT score }\end{array}$ & 3 \\
\hline Poletti et al, $2017^{61}$ & $\begin{array}{l}\text { Prospective single } \\
\text { blinded }\end{array}$ & $\begin{array}{l}\text { PIOD }=70 \\
\text { PTOL }=26\end{array}$ & Sniffin’' Sticks & $\begin{array}{l}\text { Olfactory training with } \\
\text { heavy molecular } \\
\text { weight }(\mathrm{HMW}) \text { odorant } \\
(>150 \mathrm{~g} / \mathrm{mol}, \mathrm{n}=48) \\
\text { versus low molecular } \\
\text { weight }(\mathrm{LMW}) \text { odorant } \\
(<150 \mathrm{~g} / \mathrm{mol}, \mathrm{n}=48) \text { for } \\
5 \text { months }\end{array}$ & 20 & $\begin{array}{l}\text { Overall significant } \\
\text { improvement in } \\
\text { olfaction } \\
\text { (PIOD>PTOL) } \\
\text { No difference between } \\
\text { HMW and LMW }\end{array}$ & $2 \mathrm{~B}$ \\
\hline $\begin{array}{l}\text { Konstantinidis et al, } \\
2016^{62}\end{array}$ & Prospective controlled & PIOD $=111$ & Sniffin’' Sticks & $\begin{array}{l}\text { Olfactory training (12 } \\
\text { week training Vs } 56 \\
\text { week training Vs } \\
\text { Control) }\end{array}$ & 56 & $\begin{array}{l}\text { Long term training } \\
\text { yields better function }\end{array}$ & $2 \mathrm{~B}$ \\
\hline Altundag et al, $2015^{63}$ & Prospective, controlled & $\mathrm{PIOD}=85$ & Sniffin’ Sticks & Olfactory training & 36 & $\begin{array}{l}\text { Longer olfactory } \\
\text { training with change of } \\
\text { odour was effective in } \\
\text { terms of odour } \\
\text { discrimination and } \\
\text { identification }\end{array}$ & $2 \mathrm{~B}$ \\
\hline Damm et al, $2014^{64}$ & $\begin{array}{l}\text { Prospective } \\
\text { randomised single } \\
\text { blinded controlled }\end{array}$ & PIOD $=144$ & Sniffin’ Sticks & $\begin{array}{l}\text { High concentrations of } \\
4 \text { odours Vs Very low } \\
\text { concentrations ("quasi- } \\
\text { placebo") }\end{array}$ & 38 & $\begin{array}{l}\text { Olfactory training was } \\
\text { significantly more } \\
\text { effective with high } \\
\text { concentration of odours } \\
\text { and dysfunction }<12 \\
\text { months }\end{array}$ & 2B \\
\hline Geißler et al, $2014^{65}$ & Prospective study & PIOD $=39$ & Sniffin' Sticks & $\begin{array}{l}\text { Suprathreshold } \\
\text { concentrations of } 4 \\
\text { odours }\end{array}$ & 32 & $\begin{array}{l}\text { Longer duration of } \\
\text { training ( } \geq 32 \text { weeks) } \\
\text { increased effectiveness } \\
\text { of training }\end{array}$ & $2 \mathrm{C}$ \\
\hline $\begin{array}{l}\text { Konstantinidis, et al, } \\
2013^{66}\end{array}$ & Prospective study & $\begin{array}{l}\mathrm{N}=119 \\
\mathrm{PIOD}=81\end{array}$ & Sniffin' sticks & $\begin{array}{l}\text { Olfactory training } \\
\text { group Vs control }\end{array}$ & 16 & $\begin{array}{l}\text { Significant } \\
\text { improvement training } \\
\text { groups }\end{array}$ & $2 \mathrm{C}$ \\
\hline $\begin{array}{l}\text { Kollndorfer et al } 2012 \\
67\end{array}$ & Prospective case series & $\mathrm{PIOD}=7$ & Sniffin’ Sticks & $\begin{array}{l}\text { Olfactory training with } \\
\text { 4-6 odorant twice daily }\end{array}$ & 13 & $\begin{array}{l}\text { Significant } \\
\text { improvement in }\end{array}$ & 4 \\
\hline
\end{tabular}




\begin{tabular}{|c|c|c|c|c|c|c|c|}
\hline & & & & for 12 weeks & & $\begin{array}{l}\text { threshold but no } \\
\text { change in } \\
\text { discrimination or } \\
\text { identification }\end{array}$ & \\
\hline Hummel et al $2009^{68}$ & prospective controlled & $\mathrm{N}=56 \mathrm{PIOD}=35$ & Sniffin' Sticks & $\begin{array}{l}\text { olfactory training group } \\
\text { vs no training group }\end{array}$ & 12 & $\begin{array}{l}\text { significant } \\
\text { improvement in } \\
\text { training groups with } \\
\text { average TDI scores of } \\
10.3 \text { points higher after } \\
\text { training }\end{array}$ & 3 \\
\hline Saatci et al, $2020^{69}$ & prospective controlled & PIOD $=60$ & Sniffin' Sticks & $\begin{array}{l}\text { classical olfactory } \\
\text { training group vs } \\
\text { olfactory training ball } \\
\text { group }\end{array}$ & 12 & $\begin{array}{l}\text { significant } \\
\text { improvement in OTB } \\
\text { group compared to } \\
\text { COT group }\end{array}$ & 3 \\
\hline \multicolumn{8}{|c|}{ Medical Management } \\
\hline Study & Study Design & Patients /Aetiology & $\begin{array}{l}\text { Olfactory function } \\
\text { Test }\end{array}$ & Intervention & Follow up (weeks) & Results & Level of evidence \\
\hline Nguyen et al, $2018^{70}$ & $\begin{array}{l}\text { Prospective } \\
\text { randomised controlled } \\
\text { trial }\end{array}$ & $\begin{array}{l}\mathrm{N}=133 \\
\mathrm{PIOD}=62\end{array}$ & UPSIT & $\begin{array}{l}\text { OT with saline } \\
\text { irrigation Vs OT } \\
\text { training with } \\
\text { budesonide irrigation }\end{array}$ & 32 & $\begin{array}{l}\text { Statistically significant } \\
\text { improvement in the } \\
\text { budesonide group }\end{array}$ & 1B \\
\hline Wang et al, $2018^{71}$ & $\begin{array}{l}\text { Retrospective cohort } \\
\text { study }\end{array}$ & $\begin{array}{l}\mathrm{N}=288 \\
\mathrm{PIOD}=158\end{array}$ & UPSIT & $\begin{array}{l}\text { Various bacteriostatic } \\
\text { antibiotics vs } \\
\text { Bacteriocidal vs No } \\
\text { antibiotics }\end{array}$ & 52 & $\begin{array}{l}\text { No negative effect } \\
\text { No improvement in } \\
\text { either antibiotic group } \\
\text { Odour thresholds better } \\
\text { in bactericidal group }\end{array}$ & 3 \\
\hline Whitcroft et al, $2016^{72}$ & $\begin{array}{l}\text { Prospective } \\
\text { Randomised cohort } \\
\text { Study }\end{array}$ & $\begin{array}{l}\text { Hyposmia }(\mathrm{n}=57,7 \\
\text { PIOD) }\end{array}$ & Sniffin' Sticks & $\begin{array}{l}\text { Topical sodium citrate } \\
(3.5 \mathrm{~g} / 140 \mathrm{ml}, \mathrm{pH} 7.4, \\
298 \mathrm{mOsmol} / \mathrm{L}) \text { versus } \\
\text { placebo (sodium } \\
\text { chloride) }\end{array}$ & 30 minutes & $\begin{array}{l}\text { Statistically significant } \\
\text { improvement in PVOD }\end{array}$ & $2 \mathrm{~B}$ \\
\hline Philpott et al, $2017^{73}$ & $\begin{array}{l}\text { Randomised control } \\
\text { trial }\end{array}$ & $\begin{array}{l}\mathrm{N}=55 \\
\mathrm{PIOD}=46\end{array}$ & $\begin{array}{l}\text { Threshold tests - } \\
\text { Phenyl ethyl Alcohol } \\
\text { (PEA), Acetic Acid, } \\
\text { Eucalyptol, 1-butanol }\end{array}$ & $\begin{array}{l}0.5 \mathrm{ml} \text { of } 9 \% \text { sodium } \\
\text { citrate versus placebo } \\
\text { (sterile water) }\end{array}$ & 120 minutes & $\begin{array}{l}32 \% \text { improved odour } \\
\text { sensitivity in treated } \\
\text { arm }\end{array}$ & 1B \\
\hline Whitcroft et al, $2017^{74}$ & $\begin{array}{l}\text { Randomised control } \\
\text { trial }\end{array}$ & PIOD, $\mathrm{n}=49$ & Sniffin’ Sticks & $\begin{array}{l}1 \mathrm{~mL} \text { sodium citrate } \\
\text { solution }(3.5 \mathrm{~g} / 140 \mathrm{ml}, \\
\mathrm{pH} 7.4,298 \mathrm{mOsmol} / \mathrm{L}) \\
\text { versus placebo }(1 \mathrm{~mL} \\
\text { physiological sodium } \\
\text { chloride solution })\end{array}$ & 30 minutes & $\begin{array}{l}\text { Statistically significant } \\
\text { (but not clinically } \\
\text { significant) } \\
\text { improvement in } \\
\text { composite threshold + } \\
\text { identification scores } \\
\text { following treatment } \\
\text { with sodium citrate, } \\
\text { compared with } \\
\text { placebo. }\end{array}$ & 1B \\
\hline Hummel et al, $2017^{75}$ & Retrospective cohort & $\mathrm{N}=170$ & Sniffin' Sticks & Topical Vitamin A & 45 & Significant & $2 \mathrm{~B}$ \\
\hline
\end{tabular}




\begin{tabular}{|c|c|c|c|c|c|c|c|}
\hline & study & $\mathrm{PIOD}=102$ & & $\begin{array}{l}10,000 \mathrm{IU} / \mathrm{day} \text { for } 8 \\
\text { weeks }+ \text { olfactory } \\
\text { training versus } \\
\text { olfactory training for } \\
12 \text { weeks }\end{array}$ & & $\begin{array}{l}\text { improvement in } \\
\text { Vitamin A group } \\
(37 \%)\end{array}$ & \\
\hline Henkin et al $2017^{76}$ & Prospective controlled & $\begin{array}{l}\mathrm{n}=44 \\
\mathrm{PIOD}=11\end{array}$ & $\begin{array}{l}\text { Olfactometry (odour } \\
\text { detection and } \\
\text { recognition for four } \\
\text { odours) }\end{array}$ & $\begin{array}{l}\text { Theophylline } 200- \\
800 \mathrm{mg} \text { once a day for } 2 \\
\text { to } 10 \text { months }\end{array}$ & 40 & $\begin{array}{l}\text { Increased nasal mucus } \\
\text { sonic hedgehog levels } \\
\text { associated with } \\
\text { improved detection and } \\
\text { perception of smell }\end{array}$ & 2B \\
\hline Dai et al, $2016^{77}$ & $\begin{array}{l}\text { Prospective cohort } \\
\text { study }\end{array}$ & $\begin{array}{l}\text { PIOD }(\mathrm{n}=50-\text {-Failed } \\
\text { steroid and Vitamin B } \\
\text { treatment) }\end{array}$ & UPSIT & $\begin{array}{l}\text { Traditional Chinese } \\
\text { acupuncture with } \\
\text { acupoints at the } \\
\text { nasolabial grove and } \\
\text { middle turbinates }\end{array}$ & 12 & $\begin{array}{l}\text { Improved UPSIT score } \\
\text { in TCA group from } \\
18.24 \text { to } 22.08 \\
\text { compared to the } \\
\text { observation group } \\
(17.36 \text { to } 18.64) ; \\
\text { subsequent analysis } \\
\text { dismissed these } \\
\text { findings }\end{array}$ & 2B- High risk of bias \\
\hline Kim et al, $2017^{78}$ & Retrospective study & $\begin{array}{l}\text { Olfactory dysfunction } \\
\text { ( } \mathrm{n}=491,178 \text { PIOD) }\end{array}$ & $\begin{array}{l}\text { Connecticut } \\
\text { Chemosensory Clinical } \\
\text { Research Center test } \\
\text { (threshold test) and } \\
\text { Cross-cultural Smell } \\
\text { Identification Test }\end{array}$ & $\begin{array}{l}\text { Oral prednisolone } \\
40 \mathrm{mg} \text { reducing in third } \\
\text { week by } 5 \mathrm{mg} / \mathrm{day} \mathrm{Vs} \\
\text { mometasone furoate } \\
\text { topical } 2 \text { sprays Vs } \\
\text { combination of oral } \\
\text { and topical steroid }\end{array}$ & 4 & $\begin{array}{l}59.6 \% \text { recovery in all } \\
\text { group. Combination } \\
\text { and single oral steroid } \\
\text { statistically better than } \\
\text { topical steroid alone }\end{array}$ & 4 \\
\hline Schöpf et al, $2015^{79}$ & $\begin{array}{l}\text { Prospective controlled } \\
\text { pilot study }\end{array}$ & PIOD $<10$ & Sniffin' Sticks & $\begin{array}{l}\text { Intranasal insulin } 40 \mathrm{IU} \\
(0.2 \mathrm{ml} / \text { nostril }) \mathrm{Vs} \\
\text { Saline placebo }\end{array}$ & 55 & $\begin{array}{l}\text { Immediate } \\
\text { improvement in } \\
\text { threshold and } \\
\text { discrimination. No } \\
\text { clinically significant } \\
\text { improvement. Small } \\
\text { numbers } \\
\text { Patients with higher } \\
\text { BMI performed better }\end{array}$ & 4 \\
\hline Blomqvist et al, $2003^{80}$ & $\begin{array}{l}\text { Randomised Control } \\
\text { Trial (RCT) }\end{array}$ & PIOD $(n=40)$ & $\begin{array}{l}\text { Butanol threshold } \\
\text { test }<8\end{array}$ & $\begin{array}{l}\text { 40mg of prednisolone - } \\
\text { reducing dose then } \\
\text { topical fluticasone } \\
\text { propionate for all } \\
\text { patients, then } \\
\text { randomised to placebo, } \\
\text { control and } \\
\text { continuation of } \\
\text { flucticasone } \\
\text { proprionate }\end{array}$ & 24 & $\begin{array}{l}\text { Initial 40mg of } \\
\text { prednisolone leads to } \\
\text { improvement }\end{array}$ & $2 \mathrm{~B}$ \\
\hline Henkin et al, $2009^{81}$ & $\begin{array}{l}\text { Open labelled } \\
\text { prospective study }\end{array}$ & $\begin{array}{l}\text { Multiple aetiologies } \\
\mathrm{n}=312 \text {, PIOD=97 }\end{array}$ & $\begin{array}{l}\text { Olfactometry (odour } \\
\text { detection and } \\
\text { recognition for four }\end{array}$ & $\begin{array}{l}\text { Patients who had sub- } \\
\text { optimal response to } \\
\text { oral theophylline (200- }\end{array}$ & 4 & $\begin{array}{l}\text { Statistically significant } \\
\text { improvement in } \\
\text { olfactory function in }\end{array}$ & $2 \mathrm{C}$ \\
\hline
\end{tabular}




\begin{tabular}{|c|c|c|c|c|c|c|c|}
\hline & & & odours) & $\begin{array}{l}800 \mathrm{mg} \text { ) where treated } \\
\text { with intranasal } \\
\text { theophylline } \\
\text { 20ug/day/nostril }\end{array}$ & & this subgroup & \\
\hline Reden et al, $2012^{82}$ & RCT & PIOD \& PTOL $(\mathrm{n}=54)$ & Sniffin' Sticks & $\begin{array}{l}\text { Systemic Vitamin A } \\
\text { (10,000iu capsule, once } \\
\text { a day for } 3 \text { months Vs } \\
\text { placebo) }\end{array}$ & 20 & $\begin{array}{l}\text { No statistical } \\
\text { significance in either } \\
\text { PVOD or PTOL groups }\end{array}$ & 1B \\
\hline Schriever et al, $2012^{83}$ & $\begin{array}{l}\text { Retrospective cohort } \\
\text { study }\end{array}$ & $\begin{array}{l}\text { All aetiologies }(n=425 \text {, } \\
27 \text { PIOD) }\end{array}$ & Sniffin' Sticks & $\begin{array}{l}\text { oral } \\
\text { methylprednisolone } \\
40 \mathrm{mg} \text { reducing dose for } \\
2 \text { weeks }\end{array}$ & 2 & $\begin{array}{l}\text { Statistically significant } \\
\text { improvement in } \\
\text { sniffing sticks score by } \\
6 \text { points or more }\end{array}$ & $2 \mathrm{C}$ \\
\hline Reden et al, $2011^{84}$ & RCT & PIOD, $n=55$ & Sniffin' Sticks & $\begin{array}{l}\text { 100mg BD } \\
\text { monocycline Vs } \\
\text { placebo }\end{array}$ & 28 & $\begin{array}{l}\text { No statistical difference } \\
\text { although } 15 \% \\
\text { improved in treated } \\
\text { group against } 20 \% \\
\text { spontaneously } \\
\text { improved }\end{array}$ & 1B \\
\hline Vent et al, $2010^{85}$ & Prospective study & PIOD, $n=30$ & Sniffin' Sticks & $\begin{array}{l}\text { Traditional Chinese } \\
\text { Acupuncture (TCA) } \\
\text { (The following } \\
\text { injection points were } \\
\text { chosen: DuMai } 16 \text { and } \\
20, \text { Di20, Lu } 7 \text { and } 9, \\
\text { Ma 36, and Ni3) } \\
\text { repeated weekly for } 10 \\
\text { weeks Vs Oral vitamin } \\
\text { B complex for } 12 \\
\text { weeks }\end{array}$ & 12 & $\begin{array}{l}\text { Statistical improvement } \\
\text { in TCA group }(8 / 15) \\
\text { compared to Vitamin B } \\
\text { group }(2 / 15)\end{array}$ & $2 \mathrm{C}$ \\
\hline Seo et al, $2009^{86}$ & RCT & PIOD, $n=71$ & $\begin{array}{l}\text { Butanol threshold test } \\
\text { (anosmia score } \\
\text { between } 0-3 \text { ), cross } \\
\text { culture smell } \\
\text { identification test } \\
\text { (CCSIT) }\end{array}$ & $\begin{array}{l}\text { Monotherapy } \\
\text { (prednisolone-30 mg/d } \\
\text { for the first } 3 \text { days, } 20 \\
\mathrm{mg} / \mathrm{d} \text { for } 4 \text { days and } \\
10 \mathrm{mg} / \mathrm{d} \text { for } 7 \text { days) } \\
\text { combination } \\
\text { (prednisolone/ginkgo } \\
\text { biloba- } 80 \mathrm{mg} \text { tds for } 4 \\
\text { weeks) +All given } \\
\text { mometasone furoate for } \\
\text { 4/weeks }\end{array}$ & 4 & $\begin{array}{l}\text { Statistically significant } \\
\text { improvement BTT } \\
(4.8-6.9) \text { and CCSIT }\end{array}$ & 1B- No control group \\
\hline Gudziol et al $2009^{87}$ & $\begin{array}{l}\text { Prospective } \\
\text { longitudinal pilot study }\end{array}$ & $\begin{array}{l}\mathrm{n}=19,4 \text { functional } \\
\text { hyposmia }\end{array}$ & Sniffin' Sticks & $\begin{array}{l}\text { 200mg IV or oral } \\
\text { pentoxifylline }\end{array}$ & 2days & $\begin{array}{l}\text { Increased olfactory } \\
\text { sensitivity in younger } \\
\text { patients }\end{array}$ & $2 \mathrm{C}$ \\
\hline $\begin{array}{l}\text { Fleiner and Goktas et al } \\
2011^{88}\end{array}$ & Prospective case series & $\begin{array}{l}\mathrm{N}=18 \\
\mathrm{PIOD}=8\end{array}$ & Sniffin' Sticks & $\begin{array}{l}\text { 250ug Beclomethasone } \\
\text { diproprionate spray } \\
\text { directed to olfactory } \\
\text { cleft using a specific } \\
\text { nozzle }\end{array}$ & 4 & $\begin{array}{l}2 \text { out of the } 8 \text { had } \\
\text { improved TDI }>6\end{array}$ & 4 \\
\hline
\end{tabular}




\begin{tabular}{|c|c|c|c|c|c|c|c|}
\hline Stenner et al, $2008^{89}$ & $\begin{array}{l}\text { Retrospective case } \\
\text { series }\end{array}$ & $\begin{array}{l}\mathrm{N}=89 \\
\mathrm{PIOD}=31\end{array}$ & Sniffin' Sticks & $\begin{array}{l}\text { Oral betamethasone } \\
3 \mathrm{mg} \text { followed by } \\
\text { intranasal } 1.5 \mathrm{mg} \\
\text { budesonide vs } \\
\text { intranasal } 1.5 \mathrm{mg} \\
\text { budesonide }+ \\
\text { neomycin }\end{array}$ & 12 & $\begin{array}{l}\text { Oral steroid TDI }>3 \\
\text { topical steroid alone }= \\
1 \mathrm{pt} \\
\text { steroid + neomycin } \\
2 \text { pts }\end{array}$ & 4 \\
\hline Fukazawa et al, $2005^{90}$ & Prospective study & PIOD, $n=133$ & $\begin{array}{l}\text { T\&T olfactometer and } \\
\text { VAS }\end{array}$ & $\begin{array}{l}5 \mathrm{mg} \text { intranasal } \\
\text { injection of } \\
\text { dexametasone or } \\
\text { betametasone every } 2 \\
\text { weeks for } 8 \text { weeks }\end{array}$ & 12 & $\begin{array}{l}49.6 \% \text { improvement } \\
\text { using T\&T } \\
\text { olfactometer \& visual } \\
\text { analogue scales }\end{array}$ & $2 \mathrm{C}$ \\
\hline Heilmann et al, $2004^{91}$ & Prospective study & $\begin{array}{l}\mathrm{N}=92 \\
\mathrm{PIOD}=22\end{array}$ & Sniffin' Sticks & $\begin{array}{l}\text { 40mg oral prednisolone } \\
\text { reducing dose for } 3 \\
\text { weeks Vs topical } \\
\text { Mometasone } \\
\text { Propionate for } 3 \\
\text { months }\end{array}$ & 12 & $\begin{array}{l}\text { Oral steroids improved } \\
\text { significantly. No } \\
\text { significant } \\
\text { improvement in topical }\end{array}$ & $2 \mathrm{C}$ \\
\hline Quint et al, $2002{ }^{92}$ & RCT & $\begin{array}{l}\mathrm{N}=77 \\
\mathrm{PIOD}=38\end{array}$ & $\begin{array}{l}\text { Sniffin' Sticks and } \\
\text { BTT }\end{array}$ & $\begin{array}{l}120 \mathrm{mg} / \text { day for } 4 \text { weeks } \\
\text { caroverine vs zinc } \\
\text { sulphate (control) }\end{array}$ & 4 & $\begin{array}{l}\text { Anosmic patients } \\
\text { improved but } \\
\text { significant } \\
\text { improvement in } \\
\text { hyposmic patient }\end{array}$ & 1B- \\
\hline Hummel et al. $2002^{93}$ & $\begin{array}{l}\text { Prospective clinical } \\
\text { trial }\end{array}$ & PIOD, $n=23$ & Sniffin' Sticks & $\begin{array}{l}\text { alpha lipoic acid } \\
600 \mathrm{mg} / \text { day for } 3 \text { to } 11 \\
\text { months }\end{array}$ & 16 & $\begin{array}{l}\text { Statistically significant } \\
\text { improvement in } \\
\text { olfactory function } \\
\text { especially younger } \\
\text { patients }\end{array}$ & $2 \mathrm{~B}$ \\
\hline Aiba et al, $1998^{94}$ & $\begin{array}{l}\text { Retrospective cohort } \\
\text { study }\end{array}$ & $\begin{array}{l}\mathrm{n}=426 \\
\text { PIOD, } \mathrm{n}=48\end{array}$ & Visual Analogue Scale & $\begin{array}{l}\text { 300mg zinc } \\
\text { sulphate/day for } 1 \\
\text { month VS zinc + } \\
\text { steroid (topical) + } \\
\text { vitamin b Vs top } \\
\text { steroid + Vitamin B } \\
\end{array}$ & 2 & $\begin{array}{l}\text { No significant } \\
\text { improvement in PVOD } \\
\text { group }\end{array}$ & $2 \mathrm{C}$ \\
\hline Mori et al, $1998{ }^{95}$ & Observational study & $\begin{array}{l}\mathrm{N}=889 \\
\mathrm{PIOD}=244\end{array}$ & $\begin{array}{l}\text { T\&T } \\
\text { Alinamin test / Self } \\
\text { reporting }\end{array}$ & $\begin{array}{l}\text { Topical steroids - Not } \\
\text { otherwise stated }\end{array}$ & 2-48 weeks & $\begin{array}{l}58 \% \text { improvement self- } \\
\text { reported }\end{array}$ & 4 \\
\hline Ikeda et al, $1995^{96}$ & Observational study & $\begin{array}{l}\mathrm{N}=21 \\
\mathrm{PIOD}=9\end{array}$ & $\begin{array}{l}\text { T\&T olfactometer (OT } \\
\text { \& IT) and Intravenous } \\
\text { 10mg thiamine propyl } \\
\text { disulphide }\end{array}$ & $\begin{array}{l}\text { 10-14 days of oral } \\
\text { prednisolone for PIOD } \\
\text { patients who failed to } \\
\text { improve following } \\
\text { intranasal } \\
\text { betametasone } \\
\end{array}$ & 6-12 months & No improvement & 4 \\
\hline Henkin et al, $1976^{97}$ & $\begin{array}{l}\text { Randomised double } \\
\text { blinded trial }\end{array}$ & $\begin{array}{l}\mathrm{N}=106 \\
\mathrm{PIOD}=45\end{array}$ & Olfactometry, VAS & $\begin{array}{l}\text { Zinc sulfate } 100 \mathrm{mg} \\
\text { OD } 6 / 12 \mathrm{Vs} \text { Zinc } \\
\text { sulfate } 100 \mathrm{mg} \text { daily } \\
3 / 12+\text { placebo for } 3 / 12 \\
\text { after Vs Placebo for }\end{array}$ & 24 & $\begin{array}{l}\text { No improvement in any } \\
\text { of the groups }\end{array}$ & $1 \mathrm{~A}$ \\
\hline
\end{tabular}




\begin{tabular}{|l|l|l|l|l|l|l|}
\hline & & & & $\begin{array}{l}3 / 12+\text { zinc sulfate } 100 \\
\text { mg for 3/12 Vs Placebo } \\
\text { daily for 6/12 }\end{array}$ & \\
\hline Duncan et al $1962^{98}$ & Prospective case study & $\begin{array}{l}\text { N=56 } \\
\text { PIOD=21 }\end{array}$ & Self-reporting & $\begin{array}{l}\text { Vitamin A 100,000 } \\
\text { units for 6weeks } \\
\text { followed by } 50,000- \\
15000 \text { units of oral } \\
\text { preparation for up to } \\
12 \text { weeks }\end{array}$ & 12 \\
& & & 16 patients had marked & 3 \\
improvement & \\
\hline
\end{tabular}




\section{Discussion}

\section{Conservative management}

Studies have shown that at least one third of patients presenting for evaluation of persistent PIOD had spontaneous recovery of their sense of smell without any treatment; this is in addition to the fact that many more patients will never present to a clinician due to spontaneous resolution, even if longer than the usual timecourse of an acute URTI. Reden et al, 2006 studied 262 patients with PIOD and showed a 32\% spontaneous recovery rate, although $6 \%$ of the cohort had worsening olfactory function after 14 months of follow up ${ }^{33}$. In another study of 542 patients, London et al 2007 demonstrated that regardless of aetiology, over a third of patients had spontaneous improvement of olfaction ${ }^{99}$. The rate of recovery was dependent on the degree of initial loss, age and the duration of olfactory loss ${ }^{99}$. Hence, an individualised discussion about the prognosis and likelihood of spontaneous recovery should be undertaken with all patients. Especially in the case of COVID-19 infection spontaneous recovery seems to be faster and with higher incidence and this information would be valuable to be explained in those patients. Similarly, the possible deterioration of their sense of smell without any treatment should also be explained for them to make an informed decision.

Quantification of recovery will depend on the study, but in most studies, improvement is defined as an improvement in psychophysical olfactory tests. For example in the "Sniffin Sticks" that would be a change of 5.5 points or more in the TDI score ${ }^{100}$.

\section{Olfactory Training (OT)}

There is good evidence to suggest that OT improves olfactory function in patients with PIOD. There are three meta-analyses ${ }^{101-103}$ and several prospective controlled studies that have shown improved olfactory function in patients who had olfactory training, using long term (>32 weeks), high concentration odorants. The classic OT involves a five-minute exposure to four different odorants twice a day ${ }^{68}$. These four odorants (phenyl ethyl alcohol, eucalyptol, citronellal and eugenol) are said to represent four of the six most significant odour qualities of the olfactory spectrum and have been shown to improve olfactory loss after training of 12 weeks or more.

The concept of modified olfactory training (MOT) was first published by Altundag et al $2015^{63}$ The four-odorants used in the classic OT were initially used for 12 weeks. This was followed by a second set of odorants (menthol, thyme, tangerine and jasmine) for a further 12 weeks and followed lastly by a third set of odorants (green tea, bergamot, rosemary, and gardenia) for the final 12-week period. The study was able to demonstrate better odour discrimination and identification in patients treated with the modified technique compared to the classic technique $(\mathrm{P}<0.001)$. 


\section{Smoking cessation}

Smoking has been shown to increase the prevalence and severity of olfactory dysfunction ${ }^{2,104 .}$ However, the role of smoking in olfactory loss remains a contentious issue, as other studies suggest a possible protective effect ${ }^{105}$. While the most recent meta-analysis on the topic concluded that current smoking was associated with increased risk of olfactory dysfunction ${ }^{106}$, data specifically on effects of smoking in PIOD is sparse. In a recent retrospective study, no association between smoking status and olfactory function was found in 1,313 PIOD patients ${ }^{107}$. However, as smoking cessation seems to have a positive effect on olfactory function ${ }^{108}$, this may be beneficial for some patients to undertake.

\section{Oral and Intranasal Corticosteroids}

Studies exploring the use of various formulations, routes and doses of steroid in the treatment of PIOD patients have shown some favourable outcomes ${ }^{70,78,80,86,109}$. There are however no large randomised control trials focused on PIOD patients. Nguyen et al, 2018 was able to show clinically and statistically significant $(\mathrm{p}=0.024)$ improvement in olfactory function in randomised patients treated with OT combined with budesonide irrigation compared to the control group (OT plus saline irrigation) ${ }^{70}$. It was however not clear from this study if the improvement in olfactory function after the 8-month follow up was due to spontaneous recovery or steroid effect as patients had to have had anosmia for only six months to be included in the study. It was also not clear on the method for ruling out inflammatory contribution to smell loss which would improve with the topical steroid rinses. Various comparative studies have shown improvement in olfactory function in $25-55 \%$ of patients following treatment with steroids. In a randomised control trial by Seo et al 2016, $40 \mathrm{mg}$ oral prednisolone as monotherapy or in combination with $80 \mathrm{mg}$ of ginkgo biloba for 4 weeks was shown to have significant improvement $(\mathrm{P}<0.001)$ using both BTT and CCSIT scores ${ }^{86}$. The treatment response rate was $33 \%$ in the combination therapy group compared to $14 \%$ in the monotherapy group $(\mathrm{p}=0.08)$. This study did not include a placebo or control group to ascertain if the improvement was statistically significant in comparison to an untreated group.

The question of oral versus topical steroids was evaluated by Kim et al 2017, in a retrospective study of 491 patients of which 178 had PIOD ${ }^{78}$. This study showed that the combination of oral and topical steroids or oral steroid as monotherapy significantly improves olfactory function compared to monotherapy with topical steroids and there was no significant difference between monotherapy of oral steroids or as a combination with topical steroid $(\mathrm{p}=0.978)$. Heilmann et al, 2004 demonstrated in an unblinded study, a significant improvement in PIOD patients treated with oral prednisolone $(\mathrm{p}=0.05)$ but also showed no significant improvement in the group treated with mometasone propionate ${ }^{91}$. 
It has been suggested that the favourable effect of oral corticosteroids in PIOD could be attributed to their efficacy on any underlying sinonasal inflammation ${ }^{1,110}$, possibly due to the mucosal effects of an upper respiratory tract infection; there is no definitive evidence of any additional effect on the olfactory neuroepithelium.

It has however been suggested that the technique of delivery of topical steroids may be the reason for the poor response to topical steroids; with the Kaiteki position (patients lie on the side with their head tilted and chin turned upward) allows nasal drops to reach the olfactory cleft in $96 \%$ of decongested noses and $75 \%$ in the non-decongested nose ${ }^{111}$.

Interestingly, intranasal injection of steroid has also been shown to improve significantly the olfactory function in this group of patients. In a Japanese study, Fukazawa, 2005 injected the nasal mucosa around the olfactory cleft using dexamethasone at two-weekly intervals on 8-10 occasions and was able to show $49.6 \%$ improvement in 162 patients using VAS scores. This was a non-randomised study and although T\&T was also used as an investigative tool, no results of this was provided ${ }^{90}$.

Although there is no data for post-COVID PIOD, there is increasing support for the use of corticosteroids in severe COVID to treat respiratory distress or systemic cytokine storm ${ }^{112-114}$, suggesting some potential value for managing respiratory system inflammation. Although evidence is lacking for using steroids in post-COVID PIOD, and that there is the confounding problem of steroid administration in severe disease, awareness that the treatment is safe and potentially valuable for COVID may tilt in the favour of an empirical trial for COVID PIOD ${ }^{115}$. Patients with a short history of anosmia as potentially positive in SARS-CoV2 should not take oral steroids until the full clinical presentation of the infection can be recognised. In any case the decision to initiate steroid therapy should be based on a multi-dimensional risk-benefit assessment and detailed discussion with the patient with regard to respiratory failure, considering existing co-morbidities, imaging findings and the implications of taking a short course of steroids.

\section{Non-Steroidal medical management}

Theophylline: The mechanism of action of theophylline on olfactory neuroepithelium is not fully understood. Theophylline is suggested to inhibit phosphodiesterase and increase secondary messengers such as cyclic adenosine monophosphate (cAMP) and cyclic guanosine monophosphate (cGMP) therefore aiding olfactory neuroepithelium regeneration ${ }^{81,116}$. There are no specific studies on theophylline in patients with PIOD. Henkin et al in 2009 evaluated 312 patients with hyposmia of 
multiple aetiologies treated with $200 \mathrm{mg}-800 \mathrm{mg}$ of theophylline and was able to show $50.3 \%$ of patients have statistically significant improvement in olfactory function. These patients were followed up between 6-72 months ${ }^{81}$. In 2012 the same group was also able to show improvement in olfactory function after treatment with intranasal theophylline in an unblinded pilot study ${ }^{117}$. Interpretation of these results should be viewed with caution as methodological flaws include having only been performed in one centre using non-standardised olfactory tests, multiple treatment arms and changes in treatment ${ }^{81,116 .}$

Sodium Citrate: Intranasal sodium citrate, by its ability to sequester calcium ions, is thought to reduce free mucosal calcium with subsequent reduction in negative feedback and increasing sensitivity to odorants. Whitcroft et al, 2016 performed a prospective placebo-controlled trial of monorhinal treatment of sodium citrate versus sodium chloride for patients with olfactory loss (multiple aetiologies, $\mathrm{n}=57)$ and showed improved olfactory threshold and identification only in the PIOD cohort $(n=7)^{72}$. Philpott et al, 2017 compared a single application of $0.5 \mathrm{ml}$ of $9 \%$ sodium citrate per nostril versus sterile water $(\mathrm{n}=55)$ in a randomised controlled trial and showed statistically significant improvement in olfactory function using olfactory thresholds for phenyl ethyl alcohol (PEA), 1-butanol and eucalyptol with thresholds measured up to 2 hours after intervention showing an effect lasting between 30 and 120 minutes after application ${ }^{73}$. In the latter study, the response rate was 1 in 3 of the treatment group as compared to none in the control group. More recent studies have provided mixed results ${ }^{118}$.

N-Methyl D-Aspartate (NMDA) antagonist: The mechanism of the NMDA antagonist, Caroverine on the olfactory neuroepithelium, is not entirely clear. Its mode of action is probably through inhibition of the olfactory bulb feedback mechanism. Quint et al, 2002 conducted a randomised control trial on 71 patients with non-conductive loss $(\mathrm{PIOD}=38)^{92}$. The treatment group $(\mathrm{n}=51)$ had $120 \mathrm{mg} / \mathrm{day}$ of caroverine and the control group $(n=26)$ had $140 \mathrm{mg} /$ day of zinc sulphate. The study included 38 PIOD patients and both groups were treated for 4 weeks, with the treatment group demonstrating statistically significant improvement in odour identification $(\mathrm{p}=0.042)$ and odour threshold $(\mathrm{p}=0.005)$ in patients with both anosmia and hyposmia. This therapeutic option certainly lends itself to further evaluation in a well-designed RCT.

Alpha lipoic acid (ALA): This is a fatty acid mainly used in the treatment of diabetic neuropathy, it stimulates the expression of nerve growth factors, substance $\mathrm{P}$, and neuropeptide $\mathrm{Y}$, and has antioxidative and neuroprotective capabilities. Uncontrolled prospective study of 23 patients with PIOD treated with 600mg/day of ALA for an average of 4.5 months by Hummel et al, 2002 showed at least 
moderate improvement in olfaction in $61 \%$ of the participants ${ }^{93}$.

Vitamin A: Vitamin A is known for its regenerative ability and it has been suggested that it improves olfaction by aiding regeneration of olfactory neuroepithelium. Duncan and Briggs in 1962, reported marked improvement in olfactory function in 38 of 52 patients (with mixed causes of olfactory loss), and of 16 of 21 patients with PIOD, following systemic treatment with vitamin A (50-100,000 IU per week, intramuscularly). Reden et al in 2012 however, found no improvement in olfactory function in PIOD and PTOL patients following oral administration of 10,000 IU/day of vitamin A for 3 months in a double blinded, placebo-controlled trial using Sniffin' Sticks olfactory test. More recently Hummel et al 2017, reported statistically improved olfaction in PIOD and PTOL patients in a retrospective cohort study (treatment group $n=124$, olfactory training with 10,000 IU of intranasal Vitamin A and control group, $n=46$ had olfactory training) using Sniffin' Sticks test assessment. 33\% of PIOD patients treated in this study improved compared to $23 \%$ of controls $(\mathrm{p}=0.03)^{75}$. This study however has the inherent problem of any retrospective study in that, the inability to control the differences between the groups may have confounded the results.

Minocycline: Minocycline has been shown to act as an anti-apoptotic agent which may improve olfactory function. Randomised, prospective, placebo-controlled, double-blinded study by Reden et al, 2011 using oral $50 \mathrm{mg} / \mathrm{bd}$ of minocycline versus placebo for 3 weeks found no statistical difference between the two groups ${ }^{84}$.

Zinc sulphate: All of the studies using zinc sulphate have reported no statistically significant improvement in olfactory function post-treatment, especially in PIOD ${ }^{94,95}$. Various doses have been used, ranging from $120 \mathrm{mg}$ daily to $300 \mathrm{mg}$ daily doses. Aiba et al, 1998 performed a randomised control trial with three groups (group $\mathrm{A}=$ treated with $300 \mathrm{mg}$ of oral zinc sulphate only, group B=Zinc sulphate $300 \mathrm{mg}$ and topical mometasone prioponate and group $\mathrm{C}=$ topical mometasone propionate and Vitamin B) and reported no improvement in PIOD group although the PTOL group statistically improved with zinc sulphate ${ }^{94}$. Henkin et al, 1976 also found statistically significant improvement in 45 PIOD in a randomised control trial using zinc sulphate versus placebo ${ }^{97}$. In the study by Quint $e t$ al, mentioned above on 26 patients with non-conductive loss including PIOD (81), 140mg/ day of zinc sulphate had no effect on olfactory function ${ }^{92}$. 
was able to show significant improvement in PIOD patients with traditional Chinese acupuncture $(n=15)$ compared with those treated with vitamin $B(n=15){ }^{85}$. As stated in table 1 , this study had a high risk of bias. However, a re-analysis of their data conducted by Doty et al did not support any improvement and it appears on current evidence that this treatment is not supported ${ }^{119}$.

\section{Survey of the Experts}

The survey was designed to ascertain how members of the COWoG recommend the treatment options identified from the literature review in clinical practice. Figure 2 shows the results obtained from the survey which shows clearly, an overwhelming recommendation for olfactory training and definitely no recommendation for monocycline antibiotics.

There was some discordance on oral steroids with similar number of experts likely to recommend versus similar numbers unlikely to recommend it. The consensus was that perhaps three to four days of oral steroids may be used as a diagnostic tool, in that, if the patient were to respond to this, then a conductive component may be present and a full course of steroids either for one week or a reducing regimen may prove to be useful but the risk and benefit of this need discussion with the patient. A prescription of prednisolone should be $0.5 \mathrm{mg} / \mathrm{kg}$; therefore, a typical adult would receive $40 \mathrm{mg} / \mathrm{day}$ for 1-2 weeks followed by a reducing course by $5 \mathrm{mg}$ /day over 1 week until stopping. If complete recovery occurs after 1 week of oral steroids, this should lead the clinician to reconsider the diagnosis of PIOD. The group had a similar opinion with regards to recommendation of nasal steroid as they did of oral steroids. But the side effect profile of nasal steroids being better than systemic steroids means that this perhaps will be a safer option to recommend. However, attention should be given to delivery instructions as nasal steroids are better applied in olfactory cleft area when sprayed by means of special nozzles (such as a laryngeal mucosal atomisation device) or as nasal drops in specific head positions e.g. Kaiteki position ${ }^{111}$, with the head in a dependent position.

With the exception of vitamin A, the other treatment options including theophylline, zinc sulphate, sodium citrate, caroverine, and $\alpha$-lipoic acid were all less likely to be recommended by the expert panel. Interestingly, a fair number of the experts would recommend using vitamin A drops for treatment of PIOD compared to sodium citrate. Although sodium citrate and vitamin A drops have shown promise in the treatment of PIOD, the only randomised control trial investigating the use of oral vitamin A did not show significant difference between vitamin A and placebo, although significant improvement was seen in the vitamin A group. Topical vitamin A has shown more promise but does require further RCTs 
to underpin the existing data. Sodium citrate has been shown to improve odour threshold and identification to varying degrees in the literature with the PIOD group showing the best responses.

\section{Practical challenges to Management}

1. Perceived lack of clinical importance: The negative effect of olfactory dysfunction on the quality of life of patients is not widely appreciated and is often ignored or trivialised ${ }^{13}$. This is particularly important in the COVID era, where priorities are placed on initial survival, organ impairment, and transmissibility. Various surveys of patients with olfactory dysfunction, have shown the significant morbidity associated with this disability including problems with hazard avoidance, food related problems and problems with managing odours, and social isolation ${ }^{11-13,120}$. Yet, the vast majority of clinicians do not place the same order of importance when it comes to treating this problem. An example of this is highlighted in the American Medical Association's guide to evaluation of permanent impairment which quantifies permanent olfactory loss as 1-5\% of impairment compared to deafness or visual loss which is quantified as $35 \%$ and $85 \%$ impairment respectively ${ }^{121}$.

2. Lack of testing: Most general practitioners and even otolaryngology departments do not offer standardized qualitative or quantitative assessments for patients who present with olfactory dysfunction 38 and therefore, have no way of monitoring improvements. In general, a full Ear Nose and Throat examination is performed in the clinical setting, in addition to anterior rhinoscopy, nasal endoscopy should also be performed, ideally with a $0^{\circ}$ Hopkins rod lens endoscope ( $4 \mathrm{~mm}$ diameter or smaller) to start and a $30^{\circ}$ endoscope may then be used to facilitate visualisation of the olfactory cleft ${ }^{1}$. Some patients may have MRI scans depending on their presenting symptoms, but a significant number of patients may not have any subjective or psychophysical testing which is key to offering management options ${ }^{1}$.

3. Availability of treatment: During the expert discussion, it was clear that some of the treatments discussed above were not readily available for clinical use. This therefore provides a challenge in treating patients with these drugs. The bureaucracy surrounding the ability to obtain these medications for the number of patients who present with olfactory dysfunction is increasingly making it difficult to offer treatment options for patients. Consequently, most of these treatment options are not widely used in the clinical situation. Oral and topical steroids are cheap and widely available making its use more practical in most settings. 
4. Limited evidence for available interventions: The relatively few patients accrued in the studies referenced in Table 1 underscore the challenges to date for studies of PIOD. Single institutions may be unlikely to recruit enough patients with clear OD aetiology, of recent occurrence, and appropriate enrolment criteria, to power studies placebo-controlled studies. However, the current COVID pandemic offers a rare opportunity to pursue such studies for the benefit of COVID patients, where findings may be extended to other PIOD cases in the future.

\section{Practical Guide for General practitioners}

Figure 3 below sets out a flow chart for management in primary care

1. History and examination: A thorough history should be undertaken focusing on the specifics of impairment (qualitative or quantitative, gustatory attributes (specifically distinguishing aroma from basic tastes), etc), onset, duration, any fluctuations, effects on the quality of life, medication history, past medical history, allergies, previous episodes of sinusitis, smoking, alcohol consumption (liver cirrhosis) ${ }^{122-124}$, exposure to toxins, prior significant head trauma, and family history ${ }^{125}$. If sudden onset of smell loss has occurred, then clinicians should assume that in the midst of the COVID-19 pandemic, SARS-CoV-2 infection is possible and take necessary precautions with swab testing and personal protective equipment (PPE) if face-to-face contact is needed.

2. Examination: Full head and neck examination should be carried out, noting any evidence of upper respiratory infection. Basic neurological examination should also be performed. However, we note that many GPs are now performing remote consultations where this is not feasible, but if face-to-face contact is made, then a basic nasal examination (anterior rhinoscopy) should be the minimum assessment.

3. Olfactory testing: We would consider that olfactory assessment in patients reporting olfactory dysfunction with a psychophysical test is mandatory due to the poor correlation between subjective assessment and test performance but also in order to fully determine disease burden and clinical impact of interventions ${ }^{1}$. However, we recognise that in general practice this is not usually practically possible (especially with remote consultations) and thus ideally validated questionnaires (such as the Olfactory Disorders Questionnaire $)^{36,120,126}$ should be used and if this is not possible, a recognised form of assessment, possibly quantitative and/or anchored, such as a visual analogue scale, should be used. A 
quick screening test such as the Q-Sticks test ${ }^{127}$.

4. Treatment options: Although there is limited evidence for the treatment options available, some treatment options have shown promise and may be useful in selected patients.

a. Olfactory training at home: Recent research suggests that early olfactory training aids olfactory recovery in patients with PIOD. The classical method of olfactory training may also be useful and this involves the steps described below. The classical olfactory training method involves the use 4 odorants for 12 weeks, following a rigorous schedule as described below.

Smell Training Patient Advice

The advice is not that patients "should sniff odours as often as possible". The odours for this training include rose, eucalyptus, clove and lemon.

1. Place each item into a separate bowl/jar or just take the raw material into your hands

2. Slowly and gently, inhale naturally - sniffing too quickly and deeply is likely to result in you not being able to detect anything

3. Repeat this for 20-30 seconds.

4. Move on to the next smell and repeat as above

5. Record your experience - any changes, what you notice etc, e.g. in your SmellAbility Diary Log (https://www.fifthsense.org.uk/smell-training/)

b. Steroids: As discussed above, a trial of oral steroids can aid the diagnosis of PIOD and may be used in carefully selected patients, following careful explanation of potential side effects and the limited existing data supporting its use. Diagnostic (NOT therapeutic) oral steroid trials for a limited number of days, can help to rule out concomitant residual congestion that may accompany PIOD and impair other therapeutic attempts (smell training, spontaneous recovery). Topical steroids have the benefit of a better side effect profile compared to the systemic option, hence a trial of this may be appropriate. Most GPs will defer to specialist advice rather than prescribe oral steroids, so we recognise that this may be the next step if smell training has not helped.

5. Referral to ENT specialist

6. Counselling on the hazards of smell disturbances should be undertaken at any opportunity, including advice on labelling food and gas detectors in the home environment ${ }^{12,13}$. Further help and support can be found through online fora such as the charity Fifth Sense (www.fifthsense.org.uk). 


\section{Practical Guide for Otorhinolaryngologists}

Figure 4 below sets out a flow chart for the management in secondary care

1. History and Examination: Similar to above, however it is important that all patients referred to the ENT department undergo thorough examination including anterior rhinoscopy and nasoendoscopy with rigid or flexible endoscopes to achieve complete visualisation of the olfactory cleft to exclude nasal tumours and concomitant inflammatory conditions especially when a short diagnostic trial of steroids has a positive response. Examination results should be quantified using validated clinical scoring systems where available, for comparative purposes.

2. Assessment: In patients reporting olfactory dysfunction, psychophysical olfactory assessment is mandatory in order to confirm quantitative olfactory loss and fully determine disease burden but also for the clinical impact of interventions. Where possible, validated questionnaires should be used in addition ${ }^{36,120,128}$. Where this is not possible, a recognized form of assessment, possibly quantitative and/or anchored, such as a visual analogue scale, should be used. Subjective olfactory assessment should not be undertaken in isolation, given its poor accuracy.

3. Modified olfactory training: recent evidence suggests that use of this method over a 24-36 week period is superior to performing the classical training. This training method involves the use of the classical regime for 12 weeks and then swapping to menthol, thyme tangerine, jasmine for 12 weeks, and green tea, rosemary, bergamot, gardenia for 12 weeks.

\section{Treatment options - see figure 4.}

\section{Conclusion}

The olfactory loss experienced by some patients who have been infected by the SARS-CoV-2 has brought to light the need for good evidence-based management for patients with PIOD. This review highlights the importance of olfactory training in the treatment of PIOD. The evidence for the use of medical treatment in PIOD is quite weak but it is clear that there are additional management options available for motivated patients. Oral and topical steroids may still have a role in management with Vitamin A and sodium citrate as an alternate treatment option. Non-medical options such as acupuncture need to be further investigated. The ENT research community now needs to convince 
funding bodies for the need to deliver more RCTs that can usefully inform clinicians on the place of these therapies and help to treat this much maligned group of patients. With an expectedly large number of patients with a common aetiology (COVID-19) and increasing awareness of PIOD, the opportunity for rigorous study is apparent. 


\section{References}

1. Hummel T, Whitcroft KL, Andrews P, Altundag A, Cinghi C, Costanzo RM, et al. Position paper on olfactory dysfunction. Rhinol Suppl 2017; 54:1-30.

2. Vennemann MM, Hummel T, Berger K. The association between smoking and smell and taste impairment in the general population. J Neurol 2008; 255:1121-6.

3. Landis BN, Konnerth CG, Hummel T. A study on the frequency of olfactory dysfunction. Laryngoscope 2004; 114:1764-9.

4. Brämerson A, Johansson L, Ek L, Nordin S, Bende M. Prevalence of olfactory dysfunction: the skövde population-based study. Laryngoscope 2004; 114:733-7.

5. Akerlund A, Bende M, Murphy C. Olfactory threshold and nasal mucosal changes in experimentally induced common cold. Acta Otolaryngol 1995; 115:88-92.

6. Hummel T, Rothbauer C, Barz S, Grosser K, Pauli E, Kobal G. Olfactory function in acute rhinitis. Ann N Y Acad Sci 1998; 855:616-24.

7. Pellegrino R, Walliczek-Dworschak U, Winter G, Hull D, Hummel T. Investigation of chemosensitivity during and after an acute cold. Int Forum Allergy Rhinol 2017; 7:185-91.

8. Damm M, Temmel A, Welge-Lüssen A, Eckel HE, Kreft MP, Klussmann JP, et al. [Olfactory dysfunctions. Epidemiology and therapy in Germany, Austria and Switzerland]. HNO 2004; 52:112-20.

9. Temmel AF, Quint C, Schickinger-Fischer B, Klimek L, Stoller E, Hummel T. Characteristics of olfactory disorders in relation to major causes of olfactory loss. Arch Otolaryngol Head Neck Surg 2002; 128:635-41.

10. Fonteyn S, Huart C, Deggouj N, Collet S, Eloy P, Rombaux P. Non-sinonasal-related olfactory dysfunction: A cohort of 496 patients. Eur Ann Otorhinolaryngol Head Neck Dis 2014; 131:87-91.

11. Croy I, Nordin S, Hummel T. Olfactory disorders and quality of life--an updated review. Chem Senses 2014; 39:185-94.

12. Philpott CM, Boak D. The Impact of Olfactory Disorders in the United Kingdom. Chemical Senses 2014; 39:711-8.

13. Erskine SE, Philpott CM. An unmet need: Patients with smell and taste disorders. Clin Otolaryngol 2020; 45:197-203.

14. Gerkin RC, Ohla K, Veldhuizen MG, Joseph PV, Kelly CE, Bakke AJ, et al. Recent smell loss is the best predictor of COVID-19: a preregistered, cross-sectional study. medRxiv 2020.

15. Parma V, Ohla K, Veldhuizen MG, Niv MY, Kelly CE, Bakke AJ, et al. More than smell COVID-19 is associated with severe impairment of smell, taste, and chemesthesis. Chem Senses 2020.

16. Menni C, Valdes AM, Freidin MB, Sudre CH, Nguyen LH, Drew DA, et al. Real-time tracking of self-reported symptoms to predict potential COVID-19. Nature Medicine 2020. 
17. Menni C, Valdes A, Freydin MB, Ganesh S, El-Sayed Moustafa J, Visconti A, et al. Loss of smell and taste in combination with other symptoms is a strong predictor of COVID-19 infection. medRxiv 2020:2020.04.05.20048421.

18. Rocke J, Hopkins C, Philpott C, Kumar N. Is loss of sense of smell a diagnostic marker in COVID-19: A Systematic Review and Meta-analysis. Clin Otolaryngol 2020.

19. Haehner A, Draf J, Dräger S, de With K, Hummel T. Predictive Value of Sudden Olfactory Loss in the Diagnosis of COVID-19. ORL J Otorhinolaryngol Relat Spec 2020; 82:175-80.

20. Le Bon SD, Pisarski N, Verbeke J, Prunier L, Cavelier G, Thill MP, et al. Psychophysical evaluation of chemosensory functions 5 weeks after olfactory loss due to COVID-19: a prospective cohort study on 72 patients. Eur Arch Otorhinolaryngol 2020.

21. Huart C, Philpott C, Konstantinidis I, Altundag A, Trecca EMC, Cassano M, et al. Comparison of COVID-19 and common cold chemosensory dysfunction. Rhinology 2020.

22. Butowt R, von Bartheld CS. Anosmia in COVID-19: Underlying Mechanisms and Assessment of an Olfactory Route to Brain Infection. The Neuroscientist; 0:1073858420956905.

23. Lechner M, Liu J, Counsell N, Ta N, Rocke J, Amnolsingh J, et al. Course of symptoms of loss of sense of smell over time in one thousand forty-one healthcare workers during the Covid-19 pandemic: Our experience Clinical Otolaryngology 2020; Under review.

24. Paderno A, Mattavelli D, Rampinelli V, Grammatica A, Raffetti E, Tomasoni M, et al. Olfactory and Gustatory Outcomes in COVID-19: A Prospective Evaluation in Nonhospitalized Subjects. Otolaryngol Head Neck Surg 2020:194599820939538.

25. Sayin İ, Yaşar KK, Yazici ZM. Taste and Smell Impairment in COVID-19: An AAO-HNS Anosmia Reporting Tool-Based Comparative Study. Otolaryngol Head Neck Surg 2020; 163:4739.

26. van Kempen M, Bachert C, Van Cauwenberge P. An update on the pathophysiology of rhinovirus upper respiratory tract infections. Rhinology 1999; 37:97-103.

27. Suzuki M, Saito K, Min WP, Vladau C, Toida K, Itoh H, et al. Identification of viruses in patients with postviral olfactory dysfunction. Laryngoscope 2007; 117:272-7.

28. Wang JH, Kwon HJ, Jang YJ. Detection of parainfluenza virus 3 in turbinate epithelial cells of postviral olfactory dysfunction patients. Laryngoscope 2007; 117:1445-9.

29. Yamagishi M, Fujiwara M, Nakamura H. Olfactory mucosal findings and clinical course in patients with olfactory disorders following upper respiratory viral infection. Rhinology 1994; 32:113-8.

30. Moran DT, Jafek BW, Eller PM, Rowley JC. Ultrastructural histopathology of human olfactory dysfunction. Microsc Res Tech 1992; 23:103-10.

31. Jafek BW, Murrow B, Michaels R, Restrepo D, Linschoten M. Biopsies of human olfactory epithelium. Chem Senses 2002; 27:623-8.

32. Rombaux P, Huart C, Deggouj N, Duprez T, Hummel T. Prognostic value of olfactory bulb 
volume measurement for recovery in postinfectious and posttraumatic olfactory loss. Otolaryngol Head Neck Surg 2012; 147:1136-41.

33. Reden J, Mueller A, Mueller C, Konstantinidis I, Frasnelli J, Landis BN, et al. Recovery of olfactory function following closed head injury or infections of the upper respiratory tract. Arch Otolaryngol Head Neck Surg 2006; 132:265-9.

34. Liu D, Sabha M, Damm M, Philpott C, Oleszkiewicz A, Haehner A, et al. Parosmia is associated with relevant olfactory recovery after olfactory training. Authorea, 2020.

35. Addison A, Philpott CM. A systematic review of therapeutic options for non-conductive olfactory dysfunction. The Otorhinolarnyngologist 2018; 11:61-71.

36. Langstaff L, Pradhan N, Clark A, Boak D, Salam M, Hummel T, et al. Validation of the olfactory disorders questionnaire for English-speaking patients with olfactory disorders. Clin Otolaryngol 2019; 44:715-28.

37. Soler ZM, Hyer JM, Karnezis TT, Schlosser RJ. The Olfactory Cleft Endoscopy Scale correlates with olfactory metrics in patients with chronic rhinosinusitis. Int Forum Allergy Rhinol 2016; 6:293-8.

38. Hedner M, Larsson M, Arnold N, Zucco GM, Hummel T. Cognitive factors in odor detection, odor discrimination, and odor identification tasks. Journal of Clinical and Experimental Neuropsychology 2010; 32:1062-7.

39. Gudziol V, Lötsch J, Hähner A, Zahnert T, Hummel T. Clinical significance of results from olfactory testing. Laryngoscope 2006; 116:1858-63.

40. Kobal G, Hummel T, Sekinger B, Barz S, Roscher S, Wolf S. "Sniffin' sticks": screening of olfactory performance. Rhinology 1996; 34:222-6.

41. Hummel T, Sekinger B, Wolf SR, Pauli E, Kobal G. 'Sniffin' sticks': olfactory performance assessed by the combined testing of odor identification, odor discrimination and olfactory threshold. Chem Senses 1997; 22:39-52.

42. Briner HR, Simmen D. Smell diskettes as screening test of olfaction. Rhinology 1999; $37: 145-8$.

43. Doty RL, Shaman P, Kimmelman CP, Dann MS. University of Pennsylvania Smell Identification Test: a rapid quantitative olfactory function test for the clinic. The Laryngoscope 1984; 94:176-8.

44. Cain WS, Goodspeed RB, Gent JF, Leonard G. Evaluation of olfactory dysfunction in the Connecticut Chemosensory Clinical Research Center. The Laryngoscope 1988; 98:83???8.

45. Kondo H, Matsuda T, Hashiba M, Baba S. A study of the relationship between the T\&T olfactometer and the University of Pennsylvania Smell Identification Test in a Japanese population. Am J Rhinol 1998; 12:353-8.

46. Schriever VA, Agosin E, Altundag A, Avni H, Cao Van H, Cornejo C, et al. Development of an International Odor Identification Test for Children: The Universal Sniff Test. J Pediatr 2018; 198:265-72.e3. 
47. Zou L, Dworschak A, Alizadeh R, Kamrava SK, Alwashahi M, Bock MA, et al. "U-Sniff" the international odor identification test for children: an extension of its normative database and study of global reliability. Rhinology 2020.

48. Croy I, Hoffmann H, Philpott C, Rombaux P, Welge-Luessen A, Vodicka J, et al. Retronasal testing of olfactory function: an investigation and comparison in seven countries. Eur Arch Otorhinolaryngol 2014; 271:1087-95.

49. Landis BN, Giger R, Ricchetti A, Leuchter I, Hugentobler M, Hummel T, et al. Retronasal olfactory function in nasal polyposis. Laryngoscope 2003; 113:1993-7.

50. Pfaar O, Landis BN, Frasnelli J, Huttenbrink KB, Hummel T. Mechanical obstruction of the olfactory cleft reveals differences between orthonasal and retronasal olfactory functions. Chemical Senses 2006; 31:27-31.

51. Murphy C, Nordin S, de Wijk RA, Cain WS, Polich J. Olfactory-evoked potentials: assessment of young and elderly, and comparison to psychophysical threshold. Chem Senses 1994; 19:47-56.

52. Rombaux P, Duprez T, Hummel T. Olfactory bulb volume in the clinical assessment of olfactory dysfunction. Rhinology 2009; 47:3-9.

53. Hummel T, Urbig A, Huart C, Duprez T, Rombaux P. Volume of olfactory bulb and depth of olfactory sulcus in 378 consecutive patients with olfactory loss. J Neurol 2015; 262:1046-51.

54. Zang Y, Han P, Joshi A, Hummel T. Individual variability of olfactory fMRI in normosmia and olfactory dysfunction. 2020; In press.

55. Savović J, Weeks L, Sterne JA, Turner L, Altman DG, Moher D, et al. Evaluation of the Cochrane Collaboration's tool for assessing the risk of bias in randomized trials: focus groups, online survey, proposed recommendations and their implementation. Syst Rev 2014; 3:37.

56. Moher D, Liberati A, Tetzlaff J, Altman DG, Group P. Preferred reporting items for systematic reviews and meta-analyses: the PRISMA statement. PLoS Med 2009; 6:e1000097.

57. Rudmik L, Smith TL. Development of an evidence-based review with recommendations using an online iterative process. Int Forum Allergy Rhinol 2011; 1:431-7.

58. Hummel T, Whitcroft KL, Andrews P, Altundag A, Cinghi C, Costanzo RM, et al. Position paper on olfactory dysfunction. Rhinology. Supplement 2017; 54:1-30.

59. Choi BY, Jeong H, Noh H, Park JY, Cho JH, Kim JK. Effects of Olfactory Training in Patients With Postinfectious Olfactory Dysfunction. Clin Exp Otorhinolaryngol 2020.

60. Kim BG, Kim B-Y, Shin JH, Kim SW, Kim SW. The Effect of Olfactory Training Using Korean Version Odorants: A Preliminary Study. Korean J Otorhinolaryngol-Head Neck Surg $2018 ; 61: 522-7$.

61. Poletti SC, Michel E, Hummel T. Olfactory Training Using Heavy and Light Weight Molecule Odors. Perception 2017; 46:343-51.

62. Konstantinidis I, Tsakiropoulou E, Constantinidis J. Long term effects of olfactory training in 
patients with post-infectious olfactory loss. Rhinology 2016; 54:170-5.

63. Altundag A, Cayonu M, Kayabasoglu G, Salihoglu M, Tekeli H, Saglam O, et al. Modified olfactory training in patients with postinfectious olfactory loss. Laryngoscope 2015; 125:1763-6.

64. Damm M, Pikart LK, Reimann H, Burkert S, Göktas Ö, Haxel B, et al. Olfactory training is helpful in postinfectious olfactory loss: a randomized, controlled, multicenter study. Laryngoscope 2014; 124:826-31.

65. Geißler K, Reimann H, Gudziol H, Bitter T, Guntinas-Lichius O. Olfactory training for patients with olfactory loss after upper respiratory tract infections. Eur Arch Otorhinolaryngol 2014; 271:1557-62.

66. Konstantinidis I, Tsakiropoulou E, Bekiaridou P, Kazantzidou C, Constantinidis J. Use of olfactory training in post-traumatic and postinfectious olfactory dysfunction. Laryngoscope 2013; 123:E85-90.

67. Kollndorfer K, Kowalczyk K, Hoche E, Mueller CA, Pollak M, Trattnig S, et al. Recovery of olfactory function induces neuroplasticity effects in patients with smell loss. Neural Plast 2014; 2014:140419.

68. Hummel T, Rissom K, Reden J, Hähner A, Weidenbecher M, Hüttenbrink KB. Effects of olfactory training in patients with olfactory loss. Laryngoscope 2009; 119:496-9.

69. Saatci O, Altundag A, Duz OA, Hummel T. Olfactory training ball improves adherence and olfactory outcomes in post-infectious olfactory dysfunction. Eur Arch Otorhinolaryngol 2020; 277:2125-32.

70. Nguyen TP, Patel ZM. Budesonide irrigation with olfactory training improves outcomes compared with olfactory training alone in patients with olfactory loss. Int Forum Allergy Rhinol 2018; 8:977-81.

71. Wang JJ, Chen J, Doty RL. Impact of antibiotics on smell dysfunction. World J Otorhinolaryngol Head Neck Surg 2018; 4:33-8.

72. Whitcroft KL, Merkonidis C, Cuevas M, Haehner A, Philpott C, Hummel T. Intranasal sodium citrate solution improves olfaction in post-viral hyposmia. Rhinology 2016; 54:368-74.

73. Philpott CM, Erskine SE, Clark A, Leeper A, Salam M, Sharma R, et al. A randomised controlled trial of sodium citrate spray for non-conductive olfactory disorders. Clin Otolaryngol 2017; 42:1295-302.

74. Whitcroft KL, Ezzat M, Cuevas M, Andrews P, Hummel T. The effect of intranasal sodium citrate on olfaction in post-infectious loss: results from a prospective, placebo-controlled trial in 49 patients. Clin Otolaryngol 2017; 42:557-63.

75. Hummel T, Whitcroft KL, Rueter G, Haehner A. Intranasal vitamin A is beneficial in postinfectious olfactory loss. Eur Arch Otorhinolaryngol 2017; 274:2819-25.

76. Henkin RI, Hosein S, Stateman WA, Knöppel AB, Abdelmeguid M. Improved smell function with increased nasal mucus sonic hedgehog in hyposmic patients after treatment with oral theophylline. Am J Otolaryngol 2017; 38:143-7. 
77. Dai Q, Pang Z, Yu H. Recovery of Olfactory Function in Postviral Olfactory Dysfunction Patients after Acupuncture Treatment. Evid Based Complement Alternat Med 2016;

2016:4986034.

78. Kim DH, Kim SW, Hwang SH, Kim BG, Kang JM, Cho JH, et al. Prognosis of Olfactory Dysfunction according to Etiology and Timing of Treatment. Otolaryngol Head Neck Surg 2017; 156:371-7.

79. Schöpf V, Kollndorfer K, Pollak M, Mueller CA, Freiherr J. Intranasal insulin influences the olfactory performance of patients with smell loss, dependent on the body mass index: A pilot study. Rhinology 2015; 53:371-8.

80. Blomqvist EH, Lundblad L, Bergstedt H, Stjärne P. Placebo-controlled, randomized, doubleblind study evaluating the efficacy of fluticasone propionate nasal spray for the treatment of patients with hyposmia/anosmia. Acta Otolaryngol 2003; 123:862-8.

81. Henkin RI, Velicu I, Schmidt L. An open-label controlled trial of theophylline for treatment of patients with hyposmia. Am J Med Sci 2009; 337:396-406.

82. Reden J, Lill K, Zahnert T, Haehner A, Hummel T. Olfactory function in patients with postinfectious and posttraumatic smell disorders before and after treatment with vitamin A: a double-blind, placebo-controlled, randomized clinical trial. Laryngoscope 2012; 122:1906-9.

83. Schriever VA, Lehmann S, Prange J, Hummel T. Preventing olfactory deterioration: olfactory training may be of help in older people. J Am Geriatr Soc 2014; 62:384-6.

84. Reden J, Herting B, Lill K, Kern R, Hummel T. Treatment of postinfectious olfactory disorders with minocycline: a double-blind, placebo-controlled study. Laryngoscope 2011; 121:679-82.

85. Vent J, Wang DW, Damm M. Effects of traditional Chinese acupuncture in post-viral olfactory dysfunction. Otolaryngol Head Neck Surg 2010; 142:505-9.

86. Seo BS, Lee HJ, Mo JH, Lee CH, Rhee CS, Kim JW. Treatment of postviral olfactory loss with glucocorticoids, Ginkgo biloba, and mometasone nasal spray. Arch Otolaryngol Head Neck Surg 2009; 135:1000-4.

87. Gudziol V, Hummel T. Effects of pentoxifylline on olfactory sensitivity: a postmarketing surveillance study. Arch Otolaryngol Head Neck Surg 2009; 135:291-5.

88. Fleiner F, Goktas O. Topical beclomethasone in the therapy of smelling disorders-a new application technique. Indian J Otolaryngol Head Neck Surg 2011; 63:5-9.

89. Stenner M, Vent J, Hüttenbrink KB, Hummel T, Damm M. Topical therapy in anosmia: relevance of steroid-responsiveness. Laryngoscope 2008; 118:1681-6.

90. Fukazawa K. A local steroid injection method for olfactory loss due to upper respiratory infection. Chem Senses 2005; 30 Suppl 1:i212-3.

91. Heilmann S, Huettenbrink KB, Hummel T. Local and systemic administration of corticosteroids in the treatment of olfactory loss. Am J Rhinol 2004; 18:29-33. 
92. Quint C, Temmel AF, Hummel T, Ehrenberger K. The quinoxaline derivative caroverine in the treatment of sensorineural smell disorders: a proof-of-concept study. Acta Otolaryngol 2002; 122:877-81.

93. Hummel T, Heilmann S, Hüttenbriuk KB. Lipoic acid in the treatment of smell dysfunction following viral infection of the upper respiratory tract. Laryngoscope 2002; 112:2076-80.

94. Aiba T, Sugiura M, Mori J, Matsumoto K, Tomiyama K, Okuda F, et al. Effect of zinc sulfate on sensorineural olfactory disorder. Acta Otolaryngol Suppl 1998; 538:202-4.

95. Mori J, Aiba T, Sugiura M, Matsumoto K, Tomiyama K, Okuda F, et al. Clinical study of olfactory disturbance. Acta Otolaryngol Suppl 1998; 538:197-201.

96. Ikeda K, Sakurada T, Suzaki Y, Takasaka T. Efficacy of systemic corticosteroid treatment for anosmia with nasal and paranasal sinus disease. Rhinology 1995; 33:162-5.

97. Henkin RI, Schecter PJ, Friedewald WT, Demets DL, Raff M. A double blind study of the effects of zinc sulfate on taste and smell dysfunction. Am J Med Sci 1976; 272:285-99.

98. DUNCAN RB, BRIGGS M. Treatment of uncomplicated anosmia by vitamin A. Arch Otolaryngol 1962; 75:116-24.

99. London B, Nabet B, Fisher AR, White B, Sammel MD, Doty RL. Predictors of prognosis in patients with olfactory disturbance. Ann Neurol 2008; 63:159-66.

100. Gudziol V, Lotsch J, Hahner A, Zahnert T, Hummel T. Clinical significance of results from olfactory testing. Laryngoscope 2006; 116:1858-63.

101. Sorokowska A, Drechsler E, Karwowski M, Hummel T. Effects of olfactory training: a metaanalysis. Rhinology 2017; 55:17-26.

102. Kattar N, Do TM, Unis GD, Migneron MR, Thomas AJ, McCoul ED. Olfactory Training for Postviral Olfactory Dysfunction: Systematic Review and Meta-analysis. Otolaryngol Head Neck Surg 2020:194599820943550.

103. Pekala K, Chandra RK, Turner JH. Efficacy of olfactory training in patients with olfactory loss: a systematic review and meta-analysis. Int Forum Allergy Rhinol 2016; 6:299-307.

104. Frye RE, Schwartz BS, Doty RL. Dose-related effects of cigarette smoking on olfactory function. JAMA 1990; 263:1233-6.

105. Mullol J, Alobid I, Marino-Sanchez F, Quinto L, de Haro J, Bernal-Sprekelsen M, et al. Furthering the understanding of olfaction, prevalence of loss of smell and risk factors: a population-based survey (OLFACAT study). BMJ Open 2012; 2.

106. Ajmani GS, Suh HH, Wroblewski KE, Pinto JM. Smoking and olfactory dysfunction: A systematic literature review and meta-analysis. Laryngoscope 2017; 127:1753-61.

107. Fjaeldstad AW, Ovesen T, Hummel T. The Association Between Smoking on Olfactory Dysfunction in 3,900 Patients With Olfactory Loss. Laryngoscope 2020.

108. Dinc AS, Sengezer T, Cayonu M, Sahin MM. Smoking cessation improves olfactory 
functions. Laryngoscope 2020; 130:E35-E8.

109. Schriever VA, Merkonidis C, Gupta N, Hummel C, Hummel T. Treatment of smell loss with systemic methylprednisolone. Rhinology 2012; 50:284-9.

110. Yan CH, Overdevest JB, Patel ZM. Therapeutic use of steroids in non-chronic rhinosinusitis olfactory dysfunction: a systematic evidence-based review with recommendations. Int Forum Allergy Rhinol 2019; 9:165-76.

111. Mori E, Merkonidis C, Cuevas M, Gudziol V, Matsuwaki Y, Hummel T. The administration of nasal drops in the "Kaiteki" position allows for delivery of the drug to the olfactory cleft: a pilot study in healthy subjects. Eur Arch Otorhinolaryngol 2016; 273:939-43.

112. Horby P, Lim WS, Emberson JR, Mafham M, Bell JL, Linsell L, et al. Dexamethasone in Hospitalized Patients with Covid-19 - Preliminary Report. N Engl J Med 2020.

113. McFee RB. COVID-19: Therapeutics and interventions currently under consideration. Dis Mon 2020:101058.

114. Xiang Z, Liu J, Shi D, Chen W, Li J, Yan R, et al. Glucocorticoids improve severe or critical COVID-19 by activating ACE2 and reducing IL-6 levels. Int J Biol Sci 2020; 16:2382-91.

115. Hopkins C, Alanin M, Philpott C, Harries P, Whitcroft K, Qureishi A, et al. Management of new onset loss of sense of smell during the COVID-19 pandemic - BRS Consensus Guidelines. Clin Otolaryngol 2020.

116. Henkin RI, Velicu I, Schmidt L. Relative resistance to oral theophylline treatment in patients with hyposmia manifested by decreased secretion of nasal mucus cyclic nucleotides. Am J Med Sci $2011 ; 341: 17-22$.

117. Henkin RI, Schultz M, Minnick-Poppe L. Intranasal theophylline treatment of hyposmia and hypogeusia: a pilot study. Arch Otolaryngol Head Neck Surg 2012; 138:1064-70.

118. N G. The influence of intranasally applied sodium citrate buffer on quantitative and qualitative olfactory function in patients with a post-infectious olfactory disorder. Department of Otorhinolaryngology, TU Dresden. Dresden, 2020.

119. Silas J, Doty RL. No evidence for specific benefit of acupuncture over vitamin B complex in treating persons with olfactory dysfunction. Otolaryngol Head Neck Surg 2010; 143:603; author reply -4 .

120. Frasnelli J, Hummel T. Olfactory dysfunction and daily life. Eur Arch Otorhinolaryngol 2005; 262:231-5.

121. Seabury SA, Neuhauser F, Nuckols T. American Medical Association impairment ratings and earnings losses due to disability. J Occup Environ Med 2013; 55:286-91.

122. Schubert CR, Cruickshanks KJ, Klein BE, Klein R, Nondahl DM. Olfactory impairment in older adults: five-year incidence and risk factors. Laryngoscope 2011; 121:873-8.

123. Maurage P, Callot C, Philippot P, Rombaux P, de Timary P. Chemosensory event-related potentials in alcoholism: a specific impairment for olfactory function. Biol Psychol 2011; 88:28- 
36.

124. Maurage P, Callot C, Chang B, Philippot P, Rombaux P, de Timary P. Olfactory Impairment Is Correlated with Confabulation in Alcoholism: Towards a Multimodal Testing of Orbitofrontal Cortex. Plos One 2011; 6.

125. Welge-Luessen A, Leopold D, Miwa T. Smell and Taste Disorders - Diagnostic and Clinical Work-Up. In: Welge-Luessen A, Hummel T, editors. Management of Smell and Taste Disorders A Practical Guide for Clinicians. Stuttgart: Thieme; 2013. p. 49-57.

126. Han P, Su T, Qin M, Chen H, Hummel T. A systematic review of olfactory related questionnaires and scales. Rhinology 2020.

127. Sorokowska A, Oleszkiewicz A, Minovi A, Konnerth CG, Hummel T. Fast Screening of Olfactory Function Using the Q-Sticks Test. ORL J Otorhinolaryngol Relat Spec 2019; 81:245-51.

128. Croy I, Buschhüter D, Seo HS, Negoias S, Hummel T. Individual significance of olfaction: development of a questionnaire. Eur Arch Otorhinolaryngol 2010; 267:67-71. 